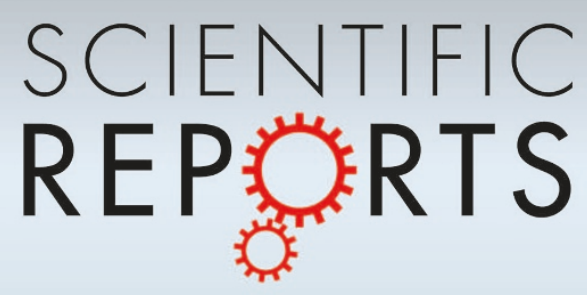

OPEN

SUBJECT AREAS:

OUTCOMES RESEARCH

CLINICAL MICROBIOLOGY

REPRODUCTIVE SIGNS AND

SYMPTOMS

Received

6 January 2014

Accepted

9 April 2014

Published

25 April 2014

Correspondence and requests for materials should be addressed to H.H. (h-honda@qg8. so-net.ne.jp)

\section{The frequent shift to intermediate flora in preterm delivery cases after abnormal vaginal flora screening}

\author{
Hiroshi Honda, Takanori Yokoyama, Yumiko Akimoto, Hirotoshi Tanimoto, Mitsue Teramoto \\ \& Hideki Teramoto
}

Department of Obstetrics and Gynecology, Hiroshima City Asa Hospital, Hiroshima, Japan.

The effect of screening and treatment for abnormal vaginal flora on the reduction of preterm deliveries remains controversial. We evaluated whether this screening and treatment reduces the preterm delivery rate for general-population pregnant women. Pregnant women of the Intervention group $(n=574)$ underwent the screening test and the treatment of vaginal metronidazole during the early second trimester, and those of the Control group $(n=1,161)$ did not. We compared the preterm delivery rate between these two groups. We also compared the profiles of vaginal flora of the preterm delivery cases with those of the pregnant women with a normal course. There was no significant difference in the preterm delivery rate between these two groups. However, in the preterm delivery cases, a frequent shift to intermediate flora was observed not before but after the screening in the Intervention group. This shift may explain why most of the previous studies failed in regard to the prevention of preterm deliveries.

$\mathrm{T}$ he prevention of preterm deliveries is still a major challenge in obstetrics in many countries. Infection is well established as one mechanism that can cause preterm deliveries. It is thought that most of the cases of intrauterine infection responsible for preterm deliveries are the result of an ascending infection. Bacterial vaginosis, which possibly triggers this ascending infection, is an imbalance of vaginal flora caused by a reduction of normally present Lactobacilli and a heavy overgrowth of mixed anaerobic bacteria. Many randomized trials have been conducted to test the hypothesis that the screening and treatment of bacterial vaginosis in pregnancy will reduce the rate of preterm deliveries, but the results have been largely negative'.

The latest Cochrane review of the antibiotics used to treat asymptomatic abnormal vaginal flora in pregnancy documented no benefit of screening and treating all pregnant women for asymptomatic abnormal vaginal flora to prevent preterm delivery ${ }^{1}$. However, this review analyzed heterogeneous trials which differ regarding the diagnosis of abnormal vaginal flora, the timing of the treatment, and the choice of antibiotics. Lamont et al. recently analyzed some homogenous studies in which oral clindamycin was used to treat pregnant women with asymptomatic abnormal vaginal flora before 22 weeks of gestation, and the authors reported positive results ${ }^{2}$. However, the efficacy of screening and treatment for abnormal vaginal flora on the reduction of preterm deliveries remains controversial.

In addition, in most of the trials, the subjects were pregnant women with asymptomatic abnormal vaginal flora, and pregnant women with normal vaginal flora were excluded from the study. If the subjects of a study are only pregnant women with asymptomatic vaginal flora, the effects of the screening and treatment on the rate of preterm delivery should be considered to be limited to only pregnant women with asymptomatic abnormal vaginal flora during the gestational weeks that served as the screening period. It is possible that some pregnant women who show normal vaginal flora during the screening period and then later have a preterm delivery experienced a shift of vaginal flora from normal to abnormal after the screening.

To the best of our knowledge, there are very few trials in which the pregnant subjects, regardless of their normal/abnormal vaginal flora, were divided into an intervention group and a control group, with the intervention group undergoing the screening for and the treatment of abnormal vaginal flora, and the control group not undergoing screening or treatment ${ }^{3}$. Both groups should include pregnant women without abnormal vaginal flora. We believe that by conducting such a between-group comparison, the precise effects of the screening and treatment of asymptomatic abnormal vaginal flora on the rate of preterm deliveries can be identified. 
The present study was a retrospective cohort study in which we compared the rates of preterm delivery between an intervention group and a control group to investigate whether the screening and treatment of abnormal vaginal flora reduces the rate of preterm deliveries in the whole population of pregnant women. We also investigated the profiles of vaginal flora taken during the screening period and on admission from pregnant women who were admitted to our hospital with a threatened preterm delivery, and we compared these profiles with those of the controls. These comparisons led to new insights into the clinical issues regarding why the screening and treatment of abnormal vaginal flora in pregnant women have failed to reduce the rate of preterm deliveries.

\section{Results}

A total of 1,975 pregnant women, 683 in service A and 1,292 in service B (see the Methods section for the details about these two services) were enrolled in the present study, and 109 pregnant women in service A and 131 pregnant women in service B were excluded as inappropriate cases for the analyses of the present study for reasons such as a history of multiple pregnancies, cervical cerclage, cervical conization, miscarriage or induced abortion before the screening period, induced preterm deliveries, and lost to follow-up (Fig. 1). Therefore, a total of 1,735 pregnant women, 574 as the Intervention group and 1,161 as the Control group, were analyzed in the present study.

Table 1 summarizes the clinical characteristics of the Intervention and Control groups. There was no significant difference in the proportion of pregnant women with risk factors related to preterm delivery between the Intervention and Control groups. In the Intervention group, the mean gestational age at the screening test for abnormal vaginal flora was $16.8 \pm 1.57$ weeks of gestation, and the mean gestational age when the administration of vaginal metronidazole was initiated for abnormal vaginal flora was $20.4 \pm 1.80$ weeks of gestation.

The profiles of vaginal flora at the screening test are summarized in Table 2. The frequency of normal flora was $67.4 \%$, that of intermediate flora was $19.0 \%$, and that of bacterial vaginosis was $13.6 \%$.

As shown in Table 3, the admission rates for threatened preterm delivery in the Intervention group and Control group were 8.36\%
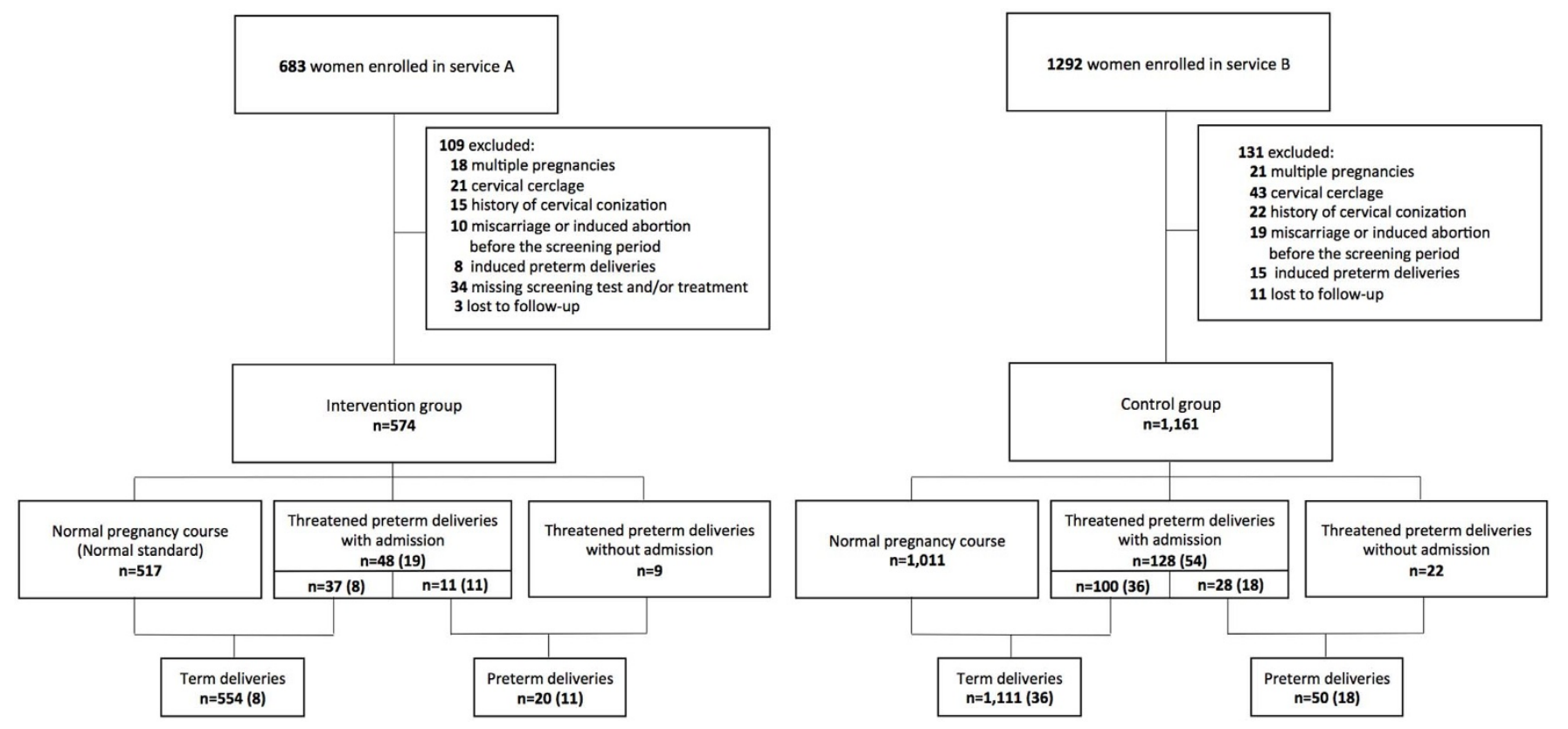

Figure 1 | Flow chart of pregnant women enrolled in the study. Of 1,975 pregnant women enrolled, the cases of 1,735 (574 in the Intervention group and 1,161 in the Control group) were analyzed. The numbers in the parentheses indicate the numbers of pregnant women whose vaginal smears were assessed on admission with a threatened preterm delivery. and $11.0 \%$, respectively, and the mean gestational ages at the admission for threatened preterm delivery of the Intervention and Control groups were $28.1 \pm 5.01$ weeks and $30.1 \pm 4.15$ weeks, respectively. There was no significant difference in the admission rate or the mean gestational age at admission between these two groups (Table 3 ). The preterm delivery rates in the Intervention group and Control group were $3.48 \%$ and $4.31 \%$, respectively, and the mean gestational ages at the preterm delivery in the Intervention and Control groups were $34.6 \pm 4.15$ weeks and $36.2 \pm 0.72$ weeks, respectively (Table 3 ). There was no significant difference in the rate or the mean gestational age at the preterm delivery between these two groups (Table 3 ).

The profiles of vaginal flora taken at the screening period from the Intervention group's subjects who were admitted with a threatened preterm delivery were not significantly different from those taken from the Normal standard subjects (Table 4). However, the profiles of vaginal flora taken on admission from the Intervention group subjects admitted with a threatened preterm delivery were significantly different from those taken from the Normal standard subjects: the proportion of the intermediate flora was significantly increased and the proportions of normal flora and bacterial vaginosis were significantly decreased compared to those of the Normal standard subjects (Table 4). In addition, the profiles of vaginal flora taken on admission from the Control group subjects admitted with a threataken from the Normal standard subjects (Table 4).

Regarding the profiles of vaginal flora taken from the pregnant women who later had a preterm delivery, those taken at the screening period from the Intervention group subjects were not significantly different from those taken from the Normal standard subjects. However, the profiles of vaginal flora taken on admission from the women with a threatened preterm delivery (from the Intervention group as well as the Control group) were significantly different from those taken from the Normal standard subjects.: the proportion of the intermediate flora was increased, and the proportions of normal flora and bacterial vaginosis were decreased compared to those of the Normal standard subjects (Table 5).

The screening test and the treatment of abnormal vaginal flora in the present study contributed to the reduction of the proportion of bacterial vaginosis, but they did not contribute to the reduction in the tened preterm delivery were not significantly different from those 
Table 1 | Clinical characteristics of the Intervention and Control groups

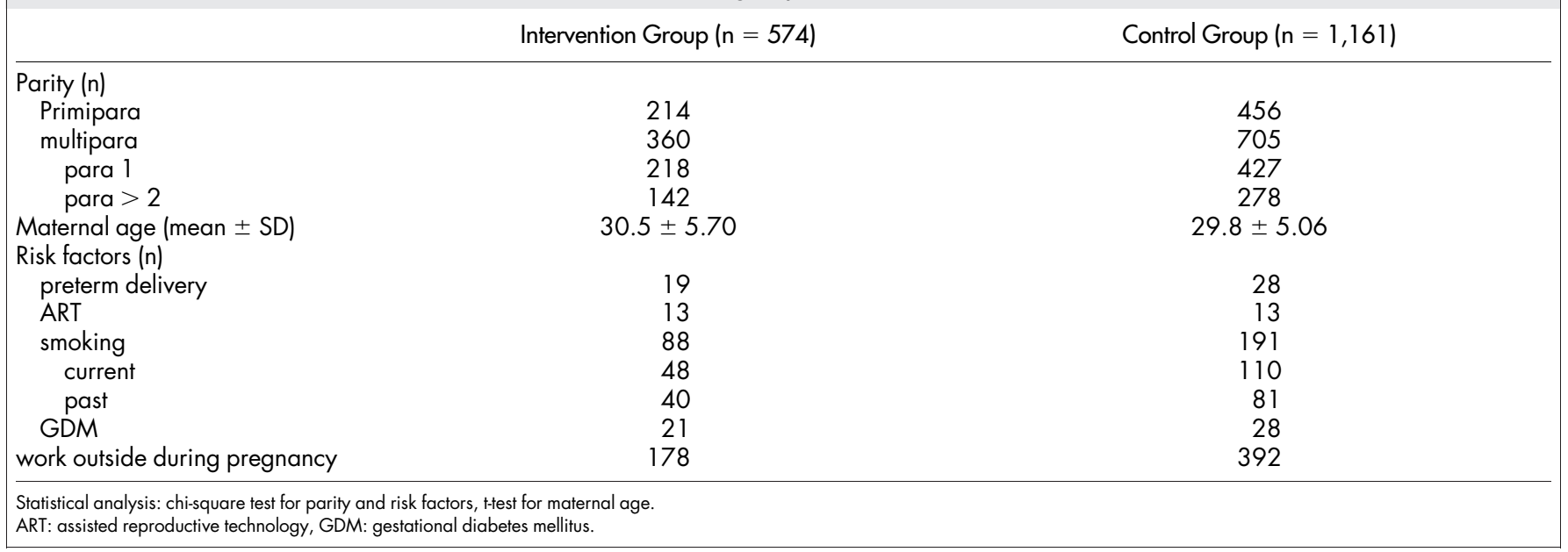

proportion of intermediate flora, or to the admission rate with a threatened preterm delivery, or to the preterm delivery rate.

\section{Discussion}

The effects of screening and treatment for abnormal vaginal flora on the desired reduction of preterm deliveries remain controversial in light of the differing results obtained in past studies ${ }^{1}$. This may be due to heterogeneity of the study protocols, which differed in a number of ways among the studies. The study populations, the timing of the screening and treatment, and the antimicrobial agents used seem to have strong impacts on the study results. We suspect that if the studies that used the same methodology regarding these issues were to be analyzed together, the results would be different from those of the previous studies.

Although the results of the present study showed a shift of vaginal flora from either normal flora or bacterial vaginosis to intermediate flora in the Intervention group women admitted with a threatened preterm delivery, this shift did not contribute to the decrease in the admission rate of threatened preterm delivery or the rate of preterm delivery. Interestingly, we observed a significant increase in the proportion of intermediate flora but not bacterial vaginosis on admission in both the Intervention group and the Control group among women who later had a preterm delivery. These findings strongly suggest that preterm delivery is associated with intermediate flora rather than bacterial vaginosis.

Donders et al. also showed that only 'partial bacterial vaginosis,' which has some overlap with intermediate flora, was significantly associated with preterm delivery ${ }^{4}$. Hay et al. ${ }^{5}$ and Donders et al. ${ }^{6}$ documented that intermediate flora is more dangerous regarding the risk of preterm delivery than bacterial vaginosis. McDonald et al. pointed out that pregnant women with an increased risk of preterm delivery have two types of abnormal vaginal flora, one consisting of predominantly bacterial vaginosis flora, and the other of aerobic vaginosis flora, in which aerobic microorganisms such as Klebsiella, E. coli, and Enterococci are present ${ }^{7}$. Cray et al. revealed that, at delivery, Klebsiella and E. coli were more often present in the

Table 2 | Profiles of the vaginal flora at the screening period for abnormal flora in the Intervention group

Nugent Score

0-3 (normal flora)

4-6 (intermediate flora)

7-10 (bacterial vaginosis) $\mathrm{n}(\%)$

$387(67.4)$

$109(19.0)$

$78(13.6)$ flora of women having a preterm delivery than in the flora of women having a term delivery8.

The antibiotic clindamycin has a broader spectrum than that of metronidazole. Clindamycin has an antimicrobial effect on aerobic microorganisms, and metronidazole lacks this effect. Lamont et al. demonstrated, in their meta-analysis, that the oral administration of clindamycin to pregnant women with asymptomatic vaginal flora only reduced the preterm delivery rate ${ }^{2}$. Brocklehurst et al. concluded on the basis of their meta-analysis that the administration of antimicrobial agents to general-population pregnant women had no effect on reducing the preterm delivery rate ${ }^{1}$. However, as in the present study, the authors of many previous studies chose metronidazole as the antimicrobial agent for the prevention of preterm deliveries. If clindamycin had been orally administered to the pregnant women with abnormal flora in the previous studies, the rate of preterm deliveries might have been reduced in those studies, as well as in the present study.

Another factor that may influence the reduction in the preterm delivery rate is the period during which the screening and the treatment of abnormal vaginal flora is performed. Lamont et al. demonstrated that the oral administration of clindamycin to pregnant women with asymptomatic vaginal flora within 22 weeks of gestation reduced the preterm delivery rate ${ }^{2}$. McDonald et al. suggested, in a Cochrane review, that the treatment of bacterial vaginosis before 20 weeks of gestation may be effective for the prevention of preterm delivery ${ }^{9}$, and they proposed that the earlier the screening and treatment of abnormal vaginal flora is performed, the better the outcome (i.e., a reduced preterm delivery rate) will be.

Many studies compared the rate of preterm delivery between an intervention group and controls with asymptomatic abnormal vaginal flora ${ }^{1}$. In these studies, therefore, the pregnant women who had normal vaginal flora during the screening period and had abnormal vaginal flora after the screening test were consequentially excluded from the subject pool to prevent preterm deliveries. Indeed, the results of the present study showed the shift of vaginal flora from normal to intermediate flora among the cases of preterm delivery after the screening test. In addition to this shift of vaginal flora, we found that there was no significant difference in the proportion of abnormal vaginal flora taken during the screening period between the cases of preterm delivery and the Normal standard in the Intervention group. Therefore, even if clindamycin is used as an antimicrobial agent for abnormal vaginal flora, it is possible that the early second-trimester screening and treatment of abnormal vaginal flora may fail in regard to the prevention of preterm delivery.

If the subjects are limited to pregnant women with asymptomatic flora, this shift would not be observed. A future study enrolling 


\begin{tabular}{|c|c|c|}
\hline & Intervention Group & Control Group \\
\hline \multirow[t]{2}{*}{$\begin{array}{l}\text { Threatened preterm delivery } \\
\mathrm{n}(\%)\end{array}$} & $48(8.36)$ & $128(11.0)$ \\
\hline & & n.s. \\
\hline \multirow[t]{2}{*}{ Mean gestational age \pm SD } & $28.1 \pm 5.01$ & $30.1 \pm 4.15$ \\
\hline & & n.s. \\
\hline \multirow{3}{*}{$\begin{array}{l}\text { Preterm delivery } \\
\mathrm{n}(\%)\end{array}$} & & \\
\hline & $20(3.48)$ & $50(4.31)$ \\
\hline & & n.s. \\
\hline \multirow[t]{2}{*}{ Mean gestational age $\pm S D$} & $34.6 \pm 4.15$ & $36.2 \pm 0.72$ \\
\hline & & n.s. \\
\hline
\end{tabular}

subjects like those in the present study must be more precise to reveal any true effect of the screening and treatment of abnormal vaginal flora on the reduction of preterm delivery compared to a study limited to pregnant women with asymptomatic vaginal flora, because we observed the frequent occurrence of a shift from normal vaginal flora among preterm delivery cases at the early second trimester to intermediate flora on admission with threatened preterm delivery. If we set the screening and the treatment of abnormal vaginal flora after this shift, we may be able to identify pregnant women at risk of preterm delivery associated with abnormal vaginal flora and thus treat them effectively.

It is also necessary to assess the profiles of vaginal flora in pregnant women with a normal course change after the screening period or at the third trimester, when the profiles of vaginal flora in preterm delivery cases were evaluated on admission in the present study. If the profiles of vaginal flora in pregnant women with a normal course do not change, the shift of vaginal flora to intermediate flora may thus be specific to preterm delivery cases.

It is well known that abnormal vaginal flora is associated with poor perinatal outcomes - in particular with an increased risk of preterm delivery $^{2,4,10-14}$. Two studies showed an $80 \%$ reduction in the rate of late miscarriage following the treatment of bacterial vaginosis ${ }^{15-17}$, and this result may support the earlier screening and treatment of abnormal vaginal flora to reduce the rate of preterm delivery. However, increasing evidence (including the present study's findings) indicates that intermediate flora is more closely associated with preterm delivery compared to bacterial vaginosis ${ }^{5,6}$, and the new finding in the present study, i.e., the shift of vaginal flora from normal to intermediate flora among the cases of preterm delivery, suggests that the choice of antimicrobial agents and the timing of the screening and the treatment of abnormal vaginal flora in pregnant women should be reconsidered.

Although the oral administration of clindamycin seems to be the most effective treatment for pregnant women with abnormal vaginal flora in regard to reducing the rate of preterm delivery, it is still unclear whether early, mid-, or late second trimester is the optimal screening period to identify pregnant women at risk of preterm delivery associated with abnormal vaginal flora. The present results suggest that the mid- or late second trimester is the better screening period to identify these women.

In two randomized controlled trials, short courses of oral metronidazole administered at mid-second trimester did not reduce the rate of preterm delivery in women with bacterial vaginosis ${ }^{18,19}$. However, this may be due to the non-use of clindamycin as the antimicrobial agent. We are thus conducting a two-step screening and treatment of abnormal vaginal flora for all pregnant women at 16 weeks and 24 weeks with oral clindamycin. Large-scale studies of general-population subjects, at least, should be performed in which the screening and the treatment of abnormal vaginal flora are done at appropriate gestational ages with oral clindamycin to clarify whether the screening and treatment of abnormal flora can reduce the rate of preterm delivery for the general population.

\section{Methods}

The medical records of the pregnant women who delivered at Hiroshima City Asa Hospital during the period from April 2009 to March 2012 were retrospectively collected for the present study, which was reviewed and approved by the Ethics Committee of Hiroshima City Asa Hospital, and was carried out in accordance with the approved guidelines of the Ethics Committee. Each woman's week of gestation was determined from the timing of the last menses and was confirmed by ultrasonography. There are two systems in our obstetrics services at Hiroshima City Asa Hospital: in one system (service A), a pregnant woman receives prenatal and perinatal care from the time of her first visit until her delivery at our hospital. In the other system (service B), until her second trimester, a pregnant woman receives prenatal care in another obstetrics setting (which offers the same obstetrical services as our hospital except for the screening test and the treatment of abnormal vaginal flora), and then from the third trimester until her delivery, she receives prenatal and perinatal care at our hospital. Every pregnant woman can freely choose either of these two services of our hospital.

Pregnant women were excluded from this study if they had any if the following: history of multiple pregnancies, cervical cerclage, cervical conization, induced preterm delivery by any complication such as pregnancy-induced hypertension, miscarriage before the screening or the treatment of abnormal vaginal flora, or having missed the screening and/or the treatment of abnormal flora at the appropriate period.

For the comparison of the pregnancy outcomes according to the differences in the screening test and the treatment of abnormal vaginal flora, we divided the pregnant women into two groups: the Intervention group, i.e., the pregnant women who participated in service A, and the Control group, i.e., the pregnant women who participated in service B.

For each woman in the Intervention group, a vaginal smear was taken in the second trimester and Gram-stained for the assessment of abnormal flora, as diagnosed by the Nugent scoring system ${ }^{20}$. The Nugent score is assessed according to the presence of large Gram-positive rods, small Gram-variable rods, and curved Gram-variable rods. Nugent scores of $0-3$ were graded as normal flora, 4-6 as intermediate flora, and 7-10 as bacterial vaginosis. The investigators who examined the vaginal smears belong to a laboratory service that is independent from our hospital's obstetrics department, and they did not know any clinical data about the pregnant women.

Women with intermediate flora or bacterial vaginosis were treated with vaginal metronidazole $(250 \mathrm{mg}$ ) for 7 days within 4 weeks after the screening test. For each woman in the Control group, both the assessment of vaginal flora and the treatment of abnormal flora in the second trimester were not done. Before the assessment of

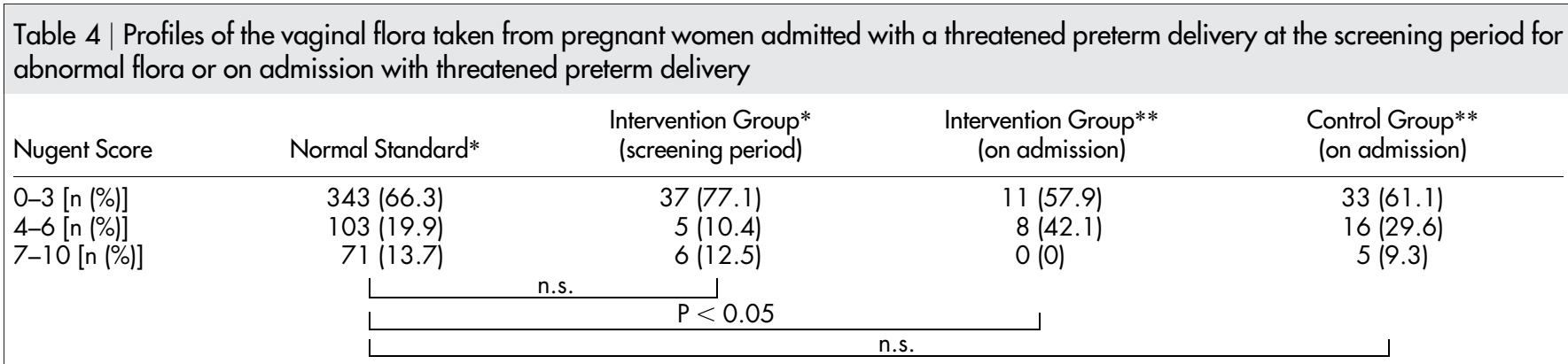

*Profiles of vaginal flora taken during the screening for abnormal vaginal flora.

**Profiles of vaginal flora taken on admission with threatened preterm delivery. The pregnant women of the Normal Standard were those of the Intervention group with no history of either admission with threatened preterm delivery or preterm delivery concerning the present pregnancy. Statistical analysis: chi-square test. n.s.: not significant. 
Table 5 | Profiles of the vaginal flora taken from pregnant women with a preterm delivery either at the screening period for abnormal vaginal flora or on admission with threatened preterm delivery

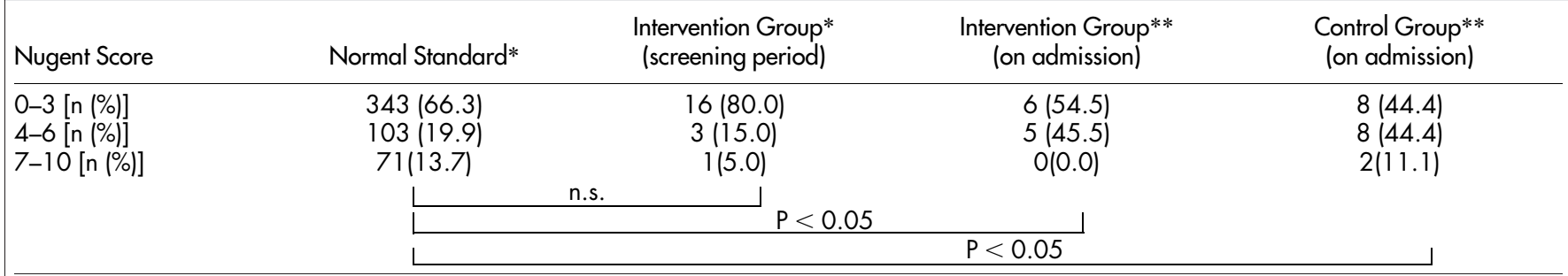

*Profiles of vaginal flora taken during the screening for abnormal vaginal flora.

**Profiles of vaginal flora taken on admission with threatened preterm delivery. The pregnant women of the Normal Standard were those of the Intervention group with no history of either admission with threatened preterm delivery or preterm delivery concerning the present pregnancy. Statistical analysis: chi-square test. n.s.: not significant.

vaginal flora either at the screening period or on admission with threatened preterm delivery, all pregnant women participating in the present study gave written informed consent.

When a pregnant woman who was enrolled in this study was hospitalized because of a threatened preterm delivery, regardless of the groups described above, her vaginal flora were assessed in the same manner as was done by the screening test in the second trimester. If the vaginal flora was assessed as either intermediate flora or bacterial vaginosis, the administration of vaginal metronidazole $(250 \mathrm{mg})$ for 7 days was initiated immediately after the diagnosis.

To determine whether the clinical backgrounds of the patients in the Intervention group and the Control group differed significantly, we used the chi-square test for comparisons of each categorical variable and the $t$-test for each continuous variable in the clinical background between the two groups. To assess the effect of the administration of vaginal metronidazole on the reduction in the rate of preterm delivery, we used the chi-square test to compare the admission rates of the pregnant women with threatened preterm deliveries and the preterm delivery rates between the Intervention and Control groups. The profiles taken from the pregnant women of the Intervention group with no history of either admission with a threatened preterm delivery or preterm delivery concerning the present pregnancy were used as the Normal standard. The profiles taken at the screening period and on admission from the Intervention group's subjects who were admitted with a threatened preterm delivery concerning the present pregnancy were compared with those of the Normal standard.

The profiles taken on admission from the Control group's subjects who were admitted with a threatened preterm delivery concerning the present pregnancy were also compared with those of the Normal standard. In addition, the profiles taken at the screening period and on admission with a threatened preterm delivery from the Intervention group's subjects who had a history of preterm delivery concerning the present pregnancy were compared with those of the Normal standard. The profiles taken on admission with a threatened preterm delivery from the Control group's subjects who had a history of preterm delivery concerning the present pregnancy were also compared with those of the Normal standard. A $p$-value $<0.05$ was considered significant.

1. Brocklehurst, P., Gordon, A., Heatley, E. \& Milan, S. J. Antibiotics for treating bacterial vaginosis in pregnancy. Cochrane Database Syst Rev. 1, CD000262 (2013).

2. Lamont, R. F. et al. Treatment of abnormal vaginal flora in early pregnancy with clindamycin for the prevention of spontaneous preterm birth: a systematic review and meta-analysis. Am J Obstet Gynecol. 205, 177-190 (2011).

3. Kiss, H., Petricevic, L., Martina, S. \& Husslein, P. Reducing the rate of preterm birth through a simple antenatal screen-and-treat programme: a retrospective cohort study. Eur J Obstet Gynecol Reprod Biol. 153, 38-42 (2010).

4. Donders, G. G. et al. Predictive value for preterm birth of abnormal vaginal flora, bacterial vaginosis and aerobic vaginitis during the first trimester of pregnancy. BJOG. 116, 1315-1324 (2009).

5. Hay, P. E. et al. Abnormal bacterial colonisation of the genital tract and subsequent preterm delivery and late miscarriage. BMJ. 308, 295-298 (1994).

6. Donders, G. G. Definition and classification of abnormal vaginal flora. Best Pract Res Clin Obstet Gynaecol. 21, 355-373 (2007).

7. McDonald, H. M., O'Loughlin, J. A., Jolley, P., Vigneswaran, R. \& McDonald, P. J. Vaginal infection and preterm labour. Br J Obstet Gynaecol. 98, 427-435 (1991).

8. Carey, J. C. \& Klebanoff, M. A. Is a change in the vaginal flora associated with an increased risk of preterm birth? Am J Obstet Gynecol. 192, 1341-1347 (2005).

9. McDonald, H., Brocklehurst, P. \& Parsons, J. Antibiotics for treating bacterial vaginosis in pregnancy. Cochrane Database Syst Rev. 1, CD000262 (2007).

10. Hay, P. E. et al. Abnormal bacterial colonisation of the genital tract and subsequent preterm delivery and late miscarriage. BMJ. 308, 295-298 (1994).
11. Hillier, S. L. et al. Association between bacterial vaginosis and preterm delivery of a low-birth-weight infant. The Vaginal Infections and Prematurity Study Group. N Engl J Med. 333, 1737-1742 (1995).

12. Kurki, T., Sivonen, A., Renkonen, O. V., Savia, E. \& Ylikorkala, O. Bacterial vaginosis in early pregnancy and pregnancy outcome. Obstet Gynecol. 80, 173-177 (1992)

13. Leitich, H. \& Kiss, H. Asymptomatic bacterial vaginosis and intermediate flora as risk factors for adverse pregnancy outcome. Best Pract Res Clin Obstet Gynaecol. 21, 375-390 (2007)

14. McGregor, J. A. et al. Cervicovaginal microflora and pregnancy outcome: results of a double-blind, placebo-controlled trial of erythromycin treatment. Am JObstet Gynecol. 163, 1580-1591 (1990).

15. Ugwumadu, A., Manyonda, I., Reid, F. \& Hay, P. Effect of early oral clindamycin on late miscarriage and preterm delivery in asymptomatic women with abnormal vaginal flora and bacterial vaginosis: a randomised controlled trial. Lancet 361, 983-988 (2003).

16. Larsson, P. G., Fåhraeus, L., Carlsson, B., Jakobsson, T. \& Forsum, U. Late miscarriage and preterm birth after treatment with clindamycin: a randomised consent design study according to Zelen.; Premature study group of the Southeast Health Care Region of Sweden. BJOG. 113, 629-637 (2006).

17. Ugwumadu, A. H. Bacterial vaginosis in pregnancy. Curr Opin Obstet Gynecol. 14, 115-118 (2002)

18. McDonald, H. M., O’Loughlin, J. A., Vigneswaran, R., Jolley, P. T. \& McDonald, P. J. Bacterial vaginosis in pregnancy and efficacy of short-course oral metronidazole treatment: a randomized controlled trial. Obstet Gynecol. $\mathbf{8 4}$ 343-348 (1994).

19. McDonald, H. M. et al. Impact of metronidazole therapy on preterm birth in women with bacterial vaginosis flora (Gardnerella vaginalis): a randomised, placebo controlled trial. Br J Obstet Gynaecol. 104, 1391-1397 (1997).

20. Nugent, R. P., Krohn, M. A. \& Hillier, S. L. Reliability of diagnosing bacterial vaginosis is improved by a standardized method of gram stain interpretation. J Clin Microbiol. 29, 297-301 (1991).

\section{Acknowledgments}

We thank Mrs. Eri Nitta and Mrs. Maki Maehara, medical clerks of Hiroshima City Asa Hospital, for the data collection in the present study.

\section{Author contributions}

H.H. conceptualized and designed the study. H.H., T.Y. and Y.A. conducted the study under the supervision of $\mathrm{H}$. Tanimoto and $\mathrm{H}$. Teramoto. H.H. wrote the initial draft of the manuscript, and $\mathrm{H}$. Tanimoto, $\mathrm{M}$. Teramoto and $\mathrm{H}$. Teramoto reviewed the manuscript. All authors read and approved the final manuscript.

\section{Additional information}

Competing financial interests: The authors declare no competing financial interests.

How to cite this article: Honda, H. et al. The frequent shift to intermediate flora in preterm delivery cases after abnormal vaginal flora screening. Sci. Rep. 4, 4799; DOI:10.1038/ srep04799 (2014).

This work is licensed under a Creative Commons Attribution-NonCommercialNoDerivs 3.0 Unported License. The images in this article are included in the article's Creative Commons license, unless indicated otherwise in the image credit; if the image is not included under the Creative Commons license, users will need to obtain permission from the license holder in order to reproduce the image. To view a copy of this license, visit http://creativecommons.org/licenses/by-nc-nd/3.0/ 